\title{
Research on the Approaches of Community-based Rehabilitation for Persons with Disability
}

\author{
You Xiaowei \\ School of Medical Sciences of Xinyu University, Xinyu, Jiangxi, China, 338000 \\ 346591653@163.com
}

Keywords: Community-based rehabilitation, Persons with disabilities, Rehabilitation services

\begin{abstract}
Rehabilitation service for people with disabilities is one of the most urgent needs of today's social service projects. This paper firstly gives the concept of community-based rehabilitation for persons with disability, then expounds the approaches of community-based rehabilitation in the foreign countries, and finally explores the approaches of community-based rehabilitation in China, including the government's purchase approach, the multiple operations approach, and the internet + approach in order to provide references for the relative researchers.
\end{abstract}

\section{Concept of Community-based Rehabilitation for Persons with Disability}

The disabled community rehabilitation services mainly provide the persons with disabilities the recovery period and late rehabilitation services as well as the prevention of disability and functional training. The community-based rehabilitation also provides the education and vocational skills, psychological counseling and other services. For persons with disabilities, this service in the form of simple goods, fast, and the economy, is conducive to their integration into the family and society, is the basis for universal rehabilitation services and the main form. The disabled community rehabilitation is one of the basic forms of rehabilitation services, compared to rehabilitation organization form, space, technology and funds are less restricted. They can use the concentrated medical resources, in various forms of rehabilitation which input minimization and effect fastest. The main forms are the basic level of rehabilitation service, home service, family rehabilitation service and neighborhood service.

The disabled community rehabilitation service is close to the primary health care. Both of them rely mainly on human resources of the community, use the community original health care or disabled grass-roots network services as far as possible, use simple suitable under the conditions of community. Both of them adjust measures to local conditions. The traditional mode of professional medical rehabilitation emphasizes the adjustment and recovery of the disabled individual function, pay attention to the individual responsibility of persons with disabilities. The technical difficulties are the high cost. Traditional medical rehabilitation model of rehabilitation limited liability in personal and family, only rely on the professional medical rehabilitation agencies to provide services, failing to reflect the participation of the main social. It does not meet the characteristics of socialization of implementation of social security, and, due to the professional rehabilitation institutions number and size limit, cannot satisfy the disabled's extensive rehabilitation needs. The implementations of social security in the perspective of society to the professional rehabilitation personnel need other subjects with the rehabilitation of the disabled mode upgrade. Community rehabilitation pattern adapted to the traditional biomedical model to biological, medical, social, psychological patterns change, pay attention to meet the needs of disabled people, to make up for 
the professional medical rehabilitation model to community rehabilitation professional organizations as the foundation to help solve the medical rehabilitation of persons with disabilities, functional training, publicity and training problems. Rehabilitation training of physical, psychological, life, education, culture and other aspects for the disabled in the community can help the persons with disabilities comprehensive rehabilitation.

\section{Approaches of Community-based Rehabilitation in the World}

\section{A: Approach of Family Service}

The mode of rehabilitation service for disabled persons in the United States is mainly family service mode, which is generally in charge of providing service by civil society organizations. They are usually the disabled and social workers put in together to form living group, become the home of the group. Each residence group of social workers according to each disabled body and ability of different formulation different rehabilitation service plans, and pay attention to personalized service, cultivate the disabled people eat the force of life ability and the team cooperation consciousness. The institutions in the USA have developed some sound policies and regulations. For example, in European countries clearly stipulates the rehabilitation needs of the disabled people can be free access to assistive technology service; provincial and county finance provides assistive technology service and product cost; all the countries, especially developed countries setting for the community rehabilitation agencies also have certain innovation. In the USA, for example, the hospital, the health center and other medical institutions are all resource centers for community rehabilitation services. Some provincial and municipal large comprehensive hospitals are also the resource centers for community rehabilitation.

\section{B: Approach of Neighborhood Service}

Germany's disabled community rehabilitation service approach is the neighborhood service. As a direct result of the good neighbor movement in Germany, the neighborhood's home is a good reflection of the basic features of the German community work. Neighborhood is an independent unit of self - management, self - management and self - financing, but its activities are partly funded by the government and subject to its supervision. The neighborhood home for the disabled community rehabilitation major daily life care, family services, and to read. Empowerment is a community rehabilitation evolution expansion reflected, refers to the rights of people with disabilities and their families in the community rehabilitation to decision-making, can grasp the community rehabilitation resources, played a leading role, and emphasizes the best by the disabled as community rehabilitation workers. Empowerment principles is disabled self-advocacy, emphasis on disabled persons in community based rehabilitation center position and continue to participate in. The things mobilize, organize and self-advocate the persons with disabilities are the collective consciousness of the disabled, not the wishes of the individual.

\section{C: Approach of Community Service}

The community service can be described as follows: Firstly, the service is the daily care for the disabled. The community establishes daily clinic, club, the old man intelligence training center and the daily care of people with disabilities health center to provide a variety of services for them. The second is the home care services to the elderly and disabled persons to provide family services or education services. Social part including interpersonal help, marriage and family, culture and art, entertainment and leisure and sports, fair, these contents are more concerned about the disabled person's inner world and spiritual, but often in the implementation in secondary status, depending to a level of economic development in the region and disabled the cause of development. Empowerment is partially including advocacy and exchanges, community mobilization, political 
participation, self-help groups and organizations of persons with disabilities, empowerment is disabled community rehabilitation principles, content is also is community rehabilitation after the development of decades of newly added content. The community rehabilitation for the development of the disabled is equally important. There are no major and secondary points. But combined with the actual situation in various regions, there can be the focus of the implementation and order.

\section{Approaches of Community-based Rehabilitation in China}

\section{A: Approach of Government's Purchase}

Government purchasing social organizations to help the disabled is a main kind of community-based rehabilitation in China. It is not only an important manifestation of the transformation of government functions, social governance mechanism innovation is more important. The State Ministry of Finance issued the opinions on the government to purchase a pilot work of the disabled service in 2013. The disabled service has become the pilot areas the government should buy. But because of the disabled social work agency has not a professional, the pilot behind the other province city. By the end of September of 2014, Huizhou City federations and dandelion disabled social service center signed a service agreement through a public bidding, marking the Huizhou city government purchase disabled home care support services work officially started. "The government, led by the CDPF and pectoral cooperation, community participation is a social work mode of the rehabilitation of the disabled. Characteristics of Huizhou pattern is according to the specific situation of Huizhou, by means of government bought station "and" the purchase of services", CDPF and the health department and other organic combination, gradually formed a community rehabilitation network of sustainable development and the socialized, and powerful ensure the community rehabilitation to specific implementation, effectively promoted the development of local community rehabilitation, truly realize the all disabled people to enjoy rehabilitation services, lay a solid foundation to achieve the equalization of opportunities for persons with disabilities and full participation in the social life of the community. Huizhou pattern relies on the support of government and the Federation of the disabled. It is mainly used to solve problems in rehabilitation of disabled persons in rural areas. The service objects are clear and the approach has some notable results.

\section{B: Approach of Multiple Operations}

The district system was set up rehabilitation center for the disabled and the disabled welfare institution was set up for the disabled persons to carry out better medical rehabilitation. Family sickbed employment guidance activities, joint civil affairs departments of education system to carry out people with disabilities of all kinds of special education learning, vocational skills training, the government through the financial sector to persons with disabilities and provide economic support. From a service providing organization, the Department of rehabilitation medicine; general hospital, specialized rehabilitation centers, community rehabilitation station is rehabilitation services provide institutions General Hospital Department of rehabilitation medicine; responsible for acute, sub-acute dysfunction rehabilitation and specialized rehabilitation center for recovery stage and late dysfunction of rehabilitation, community rehabilitation station is mainly responsible for the patients to return to the family, society after the keep training and some have not been timely rehabilitation of the late cases. The service provides training depends on the occupation rehabilitation division, and the number of China's occupation rehabilitation division is not enough to meet the rehabilitation needs of disabled persons. Therefore, looking for socialization and diversification of community rehabilitation services in the shortage of professional resources under the background of the subject is an urgent need to provide. Community rehabilitation services to provide the main body in 
addition to the community doctors, but also the families of the disabled, interested in the cause of community rehabilitation and social work volunteers, etc. Rehabilitation division of professional institutions is rehabilitation services to provide technical support and provide technical support for the entire community rehabilitation service system; community doctors are a major provider and coordinator of the community rehabilitation service. All the social forces to participate in the community rehabilitation service system play a coordinating role. The rehabilitation personnel are the carrier of the extensive development of social rehabilitation for persons with disabilities after training to master professional knowledge.

\section{C: Approach of Internet +}

Internet technology can guide the community rehabilitation service system more convenient and smart. Both of the professional and nonprofessional, the institutions and individuals can provide community-based service using the internet technology. This means that professionals should be trained for nonprofessional personnel, professional rehabilitation agencies to provide the main body of social rehabilitation services training. Two-way referral diagnosis application gateway service to "rehabilitation service system at all levels of medical institutions of two-way referral, and the establishment of regional collaborative medical data management system, realize the sharing of three stage rehabilitation medical institutions of medical resources, avoid duplication of medical, realize the patients from the onset to return to families to improve the rehabilitation treatment processes. To establish a complete system of supervision and evaluation of medical service sharing services, to establish a complete modern rehabilitation supply chain system, to realize the long-term, continuous tracking management of the service object. We can build a community cloud database, and store the mass of structured and unstructured data in the community. We can also establish the information cloud data management platform of community rehabilitation service as the transmission channel.

\section{Conclusion}

International community rehabilitation provides some advanced concepts and general principles for the development of community rehabilitation of China. Each country has its choice to implement according to the actual situation. The international organizations pull the new development of the international community of the community rehabilitation and eliminate social discrimination of persons with disabilities. However, due to the different countries, resources constrain community rehabilitation in developing countries. Chinese community rehabilitation should select the appropriate approach combined with national conditions. There is great development potential of community rehabilitation of China.

\section{References}

[1] Fu Qinglan, Fang Yufei, Lin Saijuan, The Development Process and Practices of Community Based Rehabilitation Management of the Disabled in China, Medicine and Society, 2014, 27(1): 53-55+78.

[2] Li Yan, Shi Chengli, Zhang Changjie, Chen Xiao, Significance and Developmental Status of Community-based Rehabilitation in the Disabled Rehabilitation System, Chinese Journal of Rehabilitation Theory and Practice, 2012, 18(2): 190-192.

[3] Li Zhuoya, The Study on Community-based Rehabilitation for Disabled People: Based on the Survey of Community in Guangzhou, D., Guangdong Academy of Social Sciences, 2014. 
[4] Zhang Tiantian, Tang Zuchuan, From the Participatory Development Perspective to Discuss and Analyze the Disabled Community Participation, J., Journal of Nanyang Institute of Technology, 2014, 6(5): 17-20. 